\title{
The Research of the Mechanism of Frozen Rocks' Disintegration in an Aqueous Medium (South Yakutia, Russia)
}

\author{
Nikolai N. Grib*1, Valery S. Imaev ${ }^{2,3}$, Viktor F. Rochev ${ }^{1}$ \\ ${ }^{1}$ Technical Institute (branch) North-Eastern Federal University, 67890, Neryungri (Russia) \\ ${ }^{2}$ Institute of the Earth Crust RAS, 664033 Irkutsk (Russia) \\ ${ }^{3}$ Diamond and Precious Metals Geology Institute, Siberian Division \\ Russian Academy of Science, Yakutsk, Yakutia (Russia) \\ "grib@nfygu.ru, ${ }^{2}$ imaev@crust.irk.ru
}

\begin{abstract}
The mechanism of the process of disintegration of frozen sandy-argillaceous rocks in an aqueous medium is associated with abnormal dependences of the coefficient of linear expansion of frozen dispersed rocks from the temperature and it is resulted with the following phenomenon: one can observe capillary formations in a sample, the surface of it contacting with water and water penetration in high velocity and the formation of a filtrated potential. This process is accompanied by micro and hydro impulses, cavitation, disintegrating capillary walls and the outburst of disintegrated material and appearance of natural electric field of a great value.
\end{abstract}

Keywords: dispersed rock, the coefficient of linear expansion, micro and hydro impulses, cavitation.

\section{INTRODUCTION}

Carried out experiment was aimed to make out the problem of the mechanism of disintegration of frozen sandy-argillaceous rocks in an aqueous medium, the peculiarity of which is that the disintegration of frozen rocks is higher than thawing one. It is suggested contradictory hypothesis about the mechanism of disintegration of frozen sandy-argillaceous rocks.

Some experiments took into consideration some ideas trying to find the explanation of the mechanism of disintegration process of sandy-argillaceous frozen rocks in an aqueous medium [3]:

1. The formation of thermo stresses, since they are essential till the gradient of temperature is higher at surface. However the intensity of disintegration would be more than the greater the difference of temperatures between aqueous and frozen sample. Then the disintegration would take place with the help of aggregates not with small fractions. It is not observed in described experiments [3].

2. The hypothesis of capillary pressure gives no explanation of a mechanism of disintegrated process and the reason of the intensity growth of the disintegration process while icing develops [1].

3. The ideas of hydration as the water penetration in a sample by known canals make for the growth of hydrated coating and lead to the disintegration of particle connections, these ideas also give no explanations to the reason of the growth intensity of the disintegration process when the temperature of water falls down and the minimum of dependences of parameters from the temperature and humidity exist [1].

The existing concept dealt with frozen rocks in aqueous medium does not explain the peculiarities of the given process, particularly in experiments of abnormally high intensity.

By some considerations the reason of this phenomenon are the anomalous properties of frozen clay rocks [3]. In the experiment was proved that the coefficient of linear expansion under the temperature $0<\mathrm{T}<-2 \ldots-4^{\circ} \mathrm{C}$ is negatively charged when thin dispersed rocks are heated [4].

In the experiments it was proposed the hypothesis that the mechanism of the process of disintegration of frozen sandy-argillaceous rocks in an aqueous medium is accompanied by abnormal correlations of the coefficient of linear expansion of frozen dispersed rocks from the temperature and it was resulted by 
the following phenomenon: one can observe capillary formations in a sample, the surface of it contacting with water and water penetration in high velocity and the formation of a filtrated potential (because of, first, vacuum content in the capillary; second, fresh formative surface is hydro file; third, capillary triangle section) [3]. This process is to accompanied by micro and hydro impulses, cavitation, disintegrating capillary walls and the outburst of disintegrated material.

Next, we consider the results of experiments on the verification of the hypothesis based on abnormal correlations of the coefficient of linear expansion of frozen sandy-clay rocks from the temperature.

The essence of the experiment on study of natural electrical field in the case of the process of disintegration of frozen sandy-clay rocks is the following:

1. In order of exception of humidity, granulometric content, temperature in the process of preparation all samples composed clay and sand of one granulometric content with the ratio $35 \%$ - clay, $65 \%$ sand and 30\% - humidity. The specific electrical resistance of water $\rho \mathrm{L}=60 \mathrm{Om} / \mathrm{m}$. Produced samples were frozen $\left(-17^{\circ} \mathrm{C}\right)$ and lowered to the temperature of $-3^{\circ} \mathrm{C}$ for two days.

2. The temperature of water where the samples were put was $10^{\circ} \mathrm{C}$ during the experiment.

3. The measurement of potential of own polarity was done by two ways:

a) Method of potential gradient.

Two electrodes were frozen into the sample. Electrode $+\mathrm{M}$ was put in the middle of sample, electrode $\mathrm{N}$ in one $\mathrm{cm}$ from the nearest face.

The difference of potentials of $\triangle \mathrm{UMN}$ was measured between electrodes of MN by unlimited recording during 15-16 minutes with the help of analog 4-channel registering block on a paper with automatic time-mark in $30 \mathrm{sec}$.

The registering block was graduated due graduated compensator of polarization before registration. The recording scale was $50 \mathrm{mV} / \mathrm{cm}$.

b) Method of potentials.

Electrode $+\mathrm{M}$ was frozen into the sample and electrode $-\mathrm{N}$ was at infinity, i.e. at the maximum distance from the sample and it was «earthed» in water reservoir with a sample in it.

As a result of carried out experiments it was established that the disintegration of samples of sandy clay rocks in aqueous medium is accompanied by the appearance of natural electric field in a sample.

We consider that the appearance of natural electric field to be connected with filtrated potentials resulting from a water penetration through capillary into the sample with a great velocity.

In a case of liquid flow through rocks under definite conditions filtration potentials appear. The mechanism of appearance of filtration potentials (or current potentials) in samples of frozen sandyargillaceous can be represented in the following way.

It is formed a doubled electric stratum with the liquid of the electrolyte (free unfrozen water) at the surface of capillary modeling a single rock pore. Its outside is formed by ionic diffusion the thickness of which is greater than the less the liquid concentration [2]. If the pressure difference is formed $\Delta \mathrm{P}$ between the extreme points of capillary (in a case of water immerse of frozen sandy-argillaceous sample) the liquid would take the part of ionic diffusion and the capillary itself is charged positively, and negative charge appears under great pressure at its ends.

The value of the potential of filtration through the capillary is calculated by Gelmgolts's formula:

$$
E_{f}=\frac{\varepsilon_{\iota} \rho}{4 \pi \eta} \Delta \mathrm{P}
$$

where $\rho \mathrm{L}$ - specific electrical resistance of liquid; $\xi$ - electro kinetic potential equivalent to difference of potentials at the limits of mobile and immobile parts of doubled layers and free solution; $\eta$ - liquid viscosity in the capillary; $\varepsilon \mathrm{L}$ - dielectric liquid constant. 

Russia)

The sample pores are under special consideration as capillary complex system where similar described process takes place in each of them.

Electro kinetic potential differences resulted in each sample generate electric field when the water penetrates into the frozen sample.

The magnetic intensity is determined by the expression [5]:

$E=K \varepsilon L \rho L \xi V a$,

where $\mathrm{K}$ - coefficient of the degree of rock porosity and the structure of porous space; $\mathrm{Va}$ - average velocity of liquid movement.

It is proceeded from this expression the more specific electrical resistance of filtrated solutions and velocity of filtration the more intensity of filtrating fields.

In this way exterior sample surface is negatively charged and interior is positively.

When water penetrates into sample at high velocity the maximum meaning of electrical potential of electrode at the beginning of disintegration of sample is observed (fig.1).

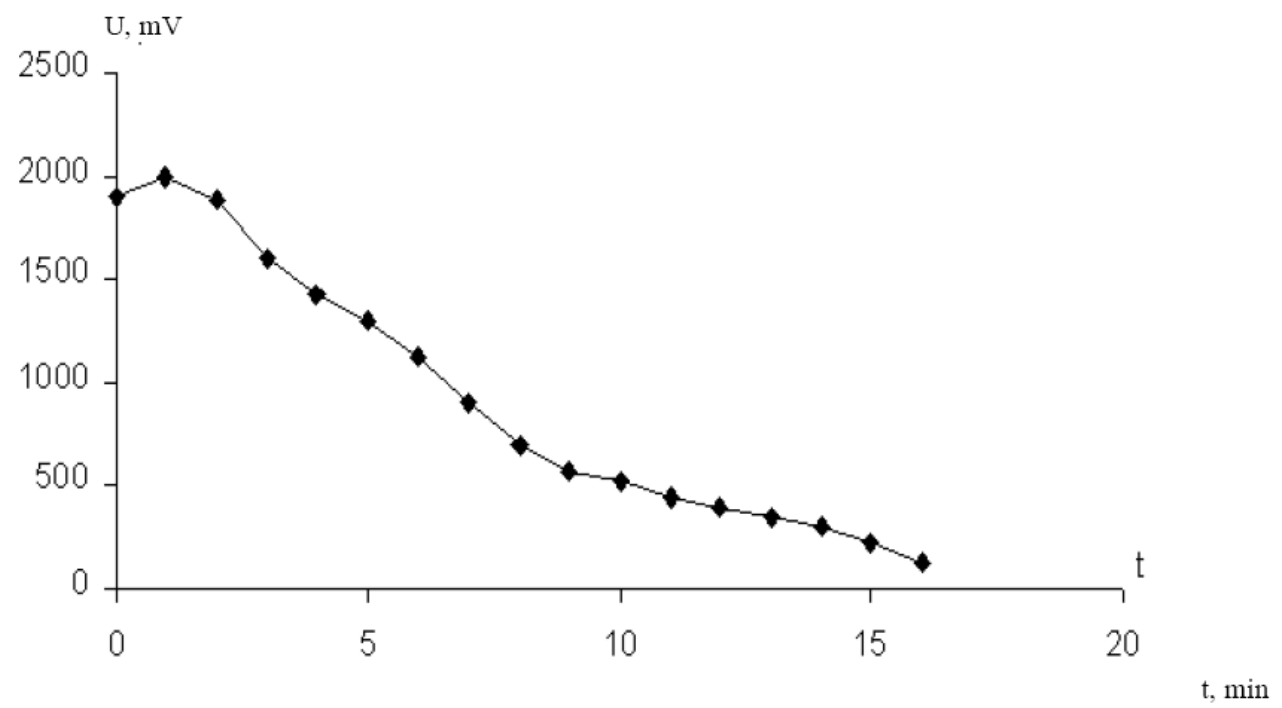

Fig1. The dependence of electrode potential $M$ of disintegrating frozen sandy-argillaceous samples in an aqueous medium from the time of disintegration measured by the potential method.

$\mathrm{UM} \cong 2000 \mathrm{mV}$, according to thawing of a sample and formation of thawed saturated bed the velocity of water penetration into a sample decreases disintegrating sample intensity and correspondingly the value of the potential UM decreases to $100 \mathrm{mV}$ properly to the equation (2).

The second run of experiments comprised the measurement of potentials used by gradient method. That is the difference between electrodes $\mathrm{M}$ and $\mathrm{N}$ in a given sample.

According to disintegration of sample takes place and it approaches to the linear of the doubled electric field to the electrode $\mathrm{N}$ the positive difference of the potential $\Delta \mathrm{UMN}$ decreases between the electrode $\mathrm{M}$ and $\mathrm{N}$ (fig. 2, table 1). When it is estimated the linear of division of solid and liquid phase of the electrode $\mathrm{N}$ the difference of potentials $\Delta \mathrm{UMN}=0$ (fig. 2, table 1).

When the electrode $\mathrm{N}$ is on the exterior side of a sample a negative potential $\Delta \mathrm{UMN}$ is to increase (fig. 2 , table 1) as the exterior side of the sample is greatly negatively charged.

When thawed sandy-argillaceous sample (with the same substantial and granulometric content and 30\% of humidity) is put into an aqueous medium by it the mechanism of disintegration and natural changes of $\Delta \mathrm{U}$ were not observed (fig 2, table 2) [3]. It is explained by the following ideas: the pores of sample are watered and there is no water penetration of high velocity in the sample. Hence it is seen some $25 \cong$ $\mathrm{mV}$ difference of potentials practically unchangeable during the experiment and due the liquid movement in saturated medium. 


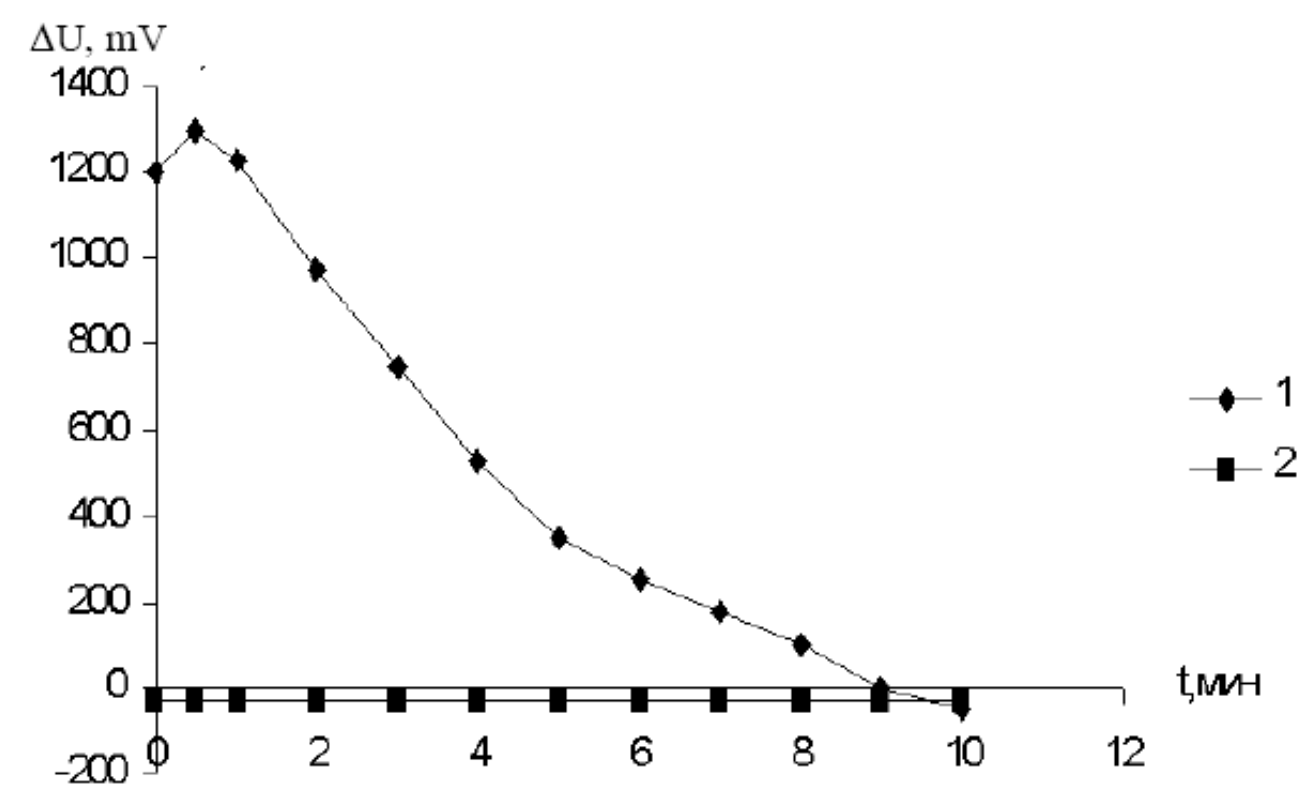

Fig2. The dependence of potential of differences $U$ of frozen (1) thawing (2) disintegrating sandy-argillaceous samples in an aqueous medium from the time of disintegration.

\section{Conclusions}

Thus, as we see completed experiments confirm the hypothesis raised by the authors Izakson V.Ju., Samohin A.V. and others, that the reason of peculiar mechanism of the process of disintegration of sandy-argillaceous rocks are abnormal dependences of the coefficient of linear expansion of frozen dispersed rocks from the temperature [3].

This work was supported by grant of Ministry of educations and Sciences of Russian Federation ( State task in the field of scientific activity N 5.1771.2014/K ) and by grant of Russian Science Foundation (RSF) N 15-17-20000

\section{REFERENCES}

[1] Bejlin A.Ju., Zaharova S.M., Shirman V.G., 1988. The technology of disintegration of highlyclay sands of gold, tin, diamond placers in an aqueous and air medium. Yakutsk, YaF SO AN SSSR Yakut Branch of the USSR Academy of Sciences] Publ. 44 p. ( in Russian)

[2] D'jakonov D.I., Leont'ev E.I., Kuznecov G.S., 1977 General course of geophysical investigations of boreholes. Moscow, Nedra Publ., 432 p. (in Russian) .

[3] Izakson V.Ju., Samohin A.V., Petrov E.E., Slepcov V.I. 1994 The items of rigidity of permafrost rock outcrops. Novosibirsk, "Science"Edition, 165 p. (in Russian).

[4] Shusherina E.P., Zajcev V.N. ,1976. The temperature deformations of permafrost dispersed rocks and reveined ices./In Permafrost investigations, N 15 Moscow, pp. 187-197. (in Russian).

[5] Jakubovskij Ju.V., 1973. Electroprospecting. Moscow, Nedra Publ.,347p. (in Russian). 


\section{AUTHORS' BIOGRAPHY}

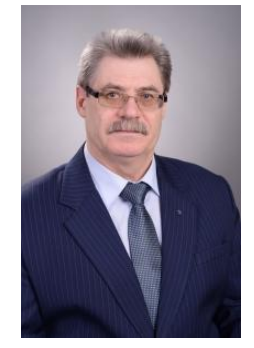

Grib Nikolay Nikolaevich - is a deputy of director of Technical institute (branch) the North-Eastern Federal University in Neruyngri sity

On the advanced study - Professor, Doctor of sciences

Area of scientific interests: studies of engineer-geological terms of coal deposits and quality of coals by geophysical methods, natural and technogenic сейсмичность.

In the advanced studies Grib Nikokay N.H the methods of decision of such issues of the day, as prognosis of indexes of quality of coals and mining and geological conditions of coal deposits, are offered from geophysical data on the stages of secret service and working mine in the conditions of Kryolitozone; he is work out technology of changeability of phisical - mechanical properties of coal-deposits breeds and on her basis a management method is worked out by explosive works on coal deposits The more than 300 advanced studies are an author and coauthor, including 8 monographs

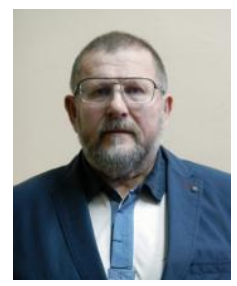

Imaev Valery Suleimanovich, chief researcher of the laboratory of earthquake engineering and seismology, Institute of Earth Crust Siberian branch of the Russian Academy of Sciences in Irkutsk sity. At the same time is a Professor at the Technical Institute (branch) of North-Eastern- Federal University (visitor-Professor part-time) Area of scientific interests - modern geological processes occurring in the large coal deposits of the North-East of Russia as well as establishing the level of seismic hazard and the change in threat level during the operation of the mine fields and underground mines in earthquake-prone areas of the Russian Arctic in scientific works. With the proposed methods In seismotectonic analysis of the territory of Yakutia and the definition of levels of seismic threat of major mineral deposits of the Republic of Sakha(Yakutia) he developed methods for studying and mapping of active faults proposed ways to reduce the potential hazard these faults $\mathrm{He}$ is the author or co-author of 250 scientific works including 7 monographs 